\title{
Multisubstituted phthalonitriles for phthalocyanine synthesis
}

\author{
Jianghong Wang, Ashot Kh. Khanamiryan and Clifford C. Leznoff*
}

Department of Chemistry, York University, Toronto, Ontario M3J IP3 Canada

\begin{abstract}
: 3,4,5,6-tetrafluorophthalonitrile and 3,4,5,6-tetrachlorophthalonitrile were used as substrates with various phenoxides to prepare 3,4,6-trihalo-5-p-substitutedphenoxyphthalonitriles, containing four substituents other than hydrogen, by nucleophilic aromatic substitution reactions. Subsequent reactions with unsymmetrical catechols gave tetrasubstitutedphthalonitriles, having four different substituents. In one instance, attempts to displace the last remaining fluoro group by an octanoxide nucleophile led to substitution of a $p$-methylphenoxy group, showing that phenoxy substituents are also labile in nucleophilic aromatic substitution reactions on phthalonitriles. Alternatively, 4,5-dimethoxyphthalonitrile or 4,5-dineopentoxyphthalonitrile underwent electrophilic aromatic substitution reactions with dibromoisocyanuric acid to give their respective 3,6 dibromophthalonitriles. Coupling of these bromophthalonitriles with tri( $n$-butyl)phenylylethynyltin and tri( $n$-butyl)vinyltin in the presence of a nickel catalyst gave 4,5-dimethoxy-3,6-bisphenylethy nylphthalonitrile, 3-bromo-4,5-dimethoxy-6-phenylethynylphthalonitrile, 4,5-dineopentoxy-3,6bisphenylethynylphthalonitrile, 3-bromo-4,5-dineopentoxy-6-phenylethynylphthalonitrile, 4,5dimethoxy-3,6-vinylphthalonitrile and 3-bromo-4,5-dimethoxy-6-vinylphthalonitrile. Reductions in the coupling steps sometimes led to 4,5-dimethoxy-3-phenylethynylphthalonitrile and 4,5-dimethoxy3-vinylphthalonitrile. Copyright (C) 2004 Society of Porphyrins \& Phthalocyanines.
\end{abstract}

KEYWORDS:phenoxyphthalonitriles,alkynylphthalonitriles, vinylphthalonitriles, dibromoisocyanuric acid, nucleophilic aromatic substitution.

\section{INTRODUCTION}

The synthesis, chemistry, properties and applications of phthalocyanines have been amply documented and reviewed $[1,2]$. The synthesis of symmetrical tetra [3-6], octa [7-9], and even hexadecaphthalocyanine (Pcs) [10-12] are relatively straightforward using an appropriately substituted phthalonitrile as the sole precursor. In most examples these phthalonitrile precursors consist of phthalonitriles containing only one substituent at the 3-or 4-position or disubstituted phthalonitriles having two identical substituents on the 4,5- or 3,6-positions, but in one unusual example at the 3,4-position [13]. For some applications, considerations of electronic, spectroscopic, or solubility are of paramount importance and more complicated phthalonitrile precursors having three or four different substitutents on a single phthalonitrile are required. These polysubstituted phthalonitriles could then be condensed to symmetrical phthalocyanines (as a mixture of regioisomers), but with each benzo group having an array of three or four different substituents. In this study two different strategies are used to prepare these desired multiply substituted phthalonitriles.

\section{EXPERIMENTAL}

Melting points (mp) were determined using a Kofler hot stage melting point apparatus and are uncorrected. Thin layer chromatography (TLC) was performed using Merck silica gel $60 \mathrm{~F}_{254}$ polyesterbacked plates and column chromatography was performed using Silicycle flash grade silica gel 
60 of particle size 40-63 $\mu \mathrm{m}$. Gel permeation chromatography was performed with Bio-Rad SX-2 Biobeads ${ }^{\star}$. All reactions were stirred with a magnetic stirrer. Ultrasound activation was carried out using a Branson 1200 sonicator. All organic solvents were dried by appropriate methods and distilled before use. Matheson high-purity argon was used to maintain inert atmosphere conditions. Infrared (IR) spectra were recorded on a Unican Mattson 3000 FT IR spectrometer using $\mathrm{KBr}$ disks. Nuclear magnetic resonance (NMR) spectra for proton and carbon were recorded on a Bruker ARX 400 high field Fourier transform instrument at room temperature. Chemical shifts are reported in ppm relative to a tetramethylsilane (TMS) internal standard. Splitting patterns of proton resonances are described as singlets (s), doublets (d), triplets (t), quartets (q), doublet of doublets (dd), multiplets (m), or as broad signals (br). Coupling constants for signals other than singlets and multiplets are reported in $\mathrm{Hz}$. Resonances are reported as the proton decoupled chemical shifts for ${ }^{13} \mathrm{C}$ NMR spectra. Ultravioletvisible (UV-vis) spectra were recorded on a HewlettPackard HP8451A Diode Array spectrophotometer. Mass spectra (MS) were recorded at $70 \mathrm{eV}$ on a VG Micromass $16 \mathrm{~F}$ mss spectrometer in the EI mode. MALDI-TOF mass spectra were performed neat using a Voyager Str instrument. Fast atom bombardment (FAB) were obtained with a Kratos Ms-50 triple analyzer mass spectrometer equipped with a FAB ion source of standard Kratos design and Ion Tech atom gun. The sample was dissolved in chloroform, and $1 \mu \mathrm{L}$ of the resulting solution added to $1 \mu \mathrm{L}$ of $m$-nitrobenzyl alcohol on the probe tip. High resolution mass spectrometric analyses (HR-MS) were performed by Dr. Alexander Young (University of Toronto, Toronto, Ontario, Canada). Microanalyses were performed by Guelph Chemical Laboratories Ltd., Guelph, Ontario.

5-p-tert-butylphenoxy-3,4,6-trifluorophthalonitrile (6). Following the procedure described for 8 below, but using 3,4,5,6-tetrafluorophthalonitrile (1), and $p$-tert-butylphenol (3), a product was obtained which was chromatographed on flash grade silica gel using ethyl acetate / hexane (3:7) to obtain an impure brown solid. Recrystallization from hexane gave white crystals of 6 in $42 \%$ yield $(0.7 \mathrm{~g}), \mathrm{mp} 125-$ $126^{\circ} \mathrm{C}$. 'H NMR (400MHz, $\mathrm{CDCl}_{3}$ ): $\delta_{\mathrm{H}}$, ppm 1.34 $(\mathrm{s}, 9 \mathrm{H}), 6.94(\mathrm{~d}, \mathrm{~J}=8 \mathrm{~Hz}, 2 \mathrm{H}), 7.40(\mathrm{~d}, \mathrm{~J}=8 \mathrm{~Hz}, 2 \mathrm{H})$. MS (EI, \%): $m / z 330\left([\mathrm{M}]^{+}, 12\right), 315$ (100). Exact mass calcd. for $\mathrm{C}_{18} \mathrm{H}_{13} \mathrm{~N}_{2} \mathrm{OF}_{3}: 330.0977$. Found: 330.0979 .

3,4,6-trifluoro-5-p-methylphenoxyphthalonitrile (7). Following the procedure described for 8 below, but using 3,4,5,6-tetrafluorophthalonitrile (1), and $p$-methylphenol (4), a product was obtained which was chromatographed on flash grade silica gel using ethyl acetate / hexane (1:1) to obtain a brown solid. Recrystallization from hexane gave white crystals $(0.96 \mathrm{~g})$ of 7 in $66.7 \%$ yield, mp $84-85^{\circ} \mathrm{C}$. 'H NMR (400 MHz, $\mathrm{CDCl}_{3}$ ): $\delta_{\mathrm{H}}$, ppm $2.38(\mathrm{~s}, 3 \mathrm{H})$, $6.91(\mathrm{~d}, \mathrm{~J}=8 \mathrm{~Hz}, 2 \mathrm{H}), 7.19(\mathrm{~d}, \mathrm{~J}=8 \mathrm{~Hz}, 2 \mathrm{H})$. MS (EI, $\%): m / z 289\left(\left[\mathrm{M}^{+}+1\right], 17\right), 288\left([\mathrm{M}]^{+}, 65\right), 91(100)$, 77 (15), 65 (49). Exact mass calcd. for $\mathrm{C}_{15} \mathrm{H}_{7} \mathrm{~N}_{2} \mathrm{OF}_{3}$ : 288.0504. Found: 288.0510 .

5-p-tert-butylphenoxy-3,4,6-trichlorophthalonitrile (8). p-tert-Butylphenol (3) (750 mg, $5 \mathrm{mmol}$ ) in dry $\mathrm{N}, \mathrm{N}$-dimethylformamide (DMF) solution (8 $\mathrm{mL})$ and $\mathrm{K}_{2} \mathrm{CO}_{3}(690 \mathrm{mg}, 5 \mathrm{mmol})$ were stirred at room temperature under argon for 30 minutes, to give a reaction in which a green color developed. 3,4,5,6-tetrachlorophthalonitrile (2) $(1.32 \mathrm{~g}, 5 \mathrm{mmol})$ was added and the reaction was maintained under anhydrous conditions for $16 \mathrm{~h}$ at $40^{\circ} \mathrm{C}$. The solvent was then removed by rotatory evaporation at $60^{\circ} \mathrm{C}$. The residue was suspended in ethyl acetate, suction filtered through Celite, and the solvent was removed from the filtrate in vacuo at $40^{\circ} \mathrm{C}$. The resulting brown residue was chromatographed on flash grade silica gel using ethyl acetate / hexane (1:4) as the eluting solvents to get a powder. Recrystallization from hexane gave white crystals ( $810 \mathrm{mg}$, $43 \%$ yield), mp 95-97 ${ }^{\circ} \mathrm{C}$. ' $\mathrm{H}$ NMR (400 MHz, $\mathrm{CDCl}_{3}$ ): $\delta_{\mathrm{H}}, \mathrm{ppm}$ $1.33(\mathrm{~s}, 9 \mathrm{H}), 6.75(\mathrm{~d}, \mathrm{~J}=7.4 \mathrm{~Hz}, 2 \mathrm{H}), 7.36(\mathrm{~d}, \mathrm{~J}=7.4$ $\mathrm{Hz}, 2 \mathrm{H})$. MS (EI, \%): $m / z 378\left([\mathrm{M}]^{+}, 18\right), 380\left(\left[\mathrm{M}^{+}\right.\right.$ $+2], 18), 363(100), 365$ (98). Exact mass calcd. for $\mathrm{C}_{18} \mathrm{H}_{13} \mathrm{~N}_{2} \mathrm{OCl}_{3}: 378.0092$. Found: 378.0093 .

3,4,6-trichloro-5-p-methylphenoxyphthalonitrile (9). Following the procedure described for 8 above, but using 3,4,5,6-tetrachlorophthalonitrile (2), and $p$-methylphenol (4), a product was obtained which was chromatographed on flash grade silica gel using ethyl acetate / hexane (1:9) to get a light brown liquid, which slowly crystallized. Recrystallization from ethyl acetate / hexane gave crystals of $9(208$ $\mathrm{mg}, 62 \%$ ), mp $167-169^{\circ} \mathrm{C}$. 'H NMR (400 MHz, $\left.\mathrm{CDCl}_{3}\right): \delta_{\mathrm{H}}, \operatorname{ppm} 2.35(\mathrm{~s}, 3 \mathrm{H}), 6.72(\mathrm{~d}, \mathrm{~J}=8.1 \mathrm{~Hz}$, $2 \mathrm{H}), 7.15-7.17(\mathrm{~d}, \mathrm{~J}=8.1 \mathrm{~Hz}, 2 \mathrm{H}) . \mathrm{MS}(\mathrm{EI}, \%): \mathrm{m} / \mathrm{z}$ $336\left([\mathrm{M}]^{+}, 82\right), 338\left(\left[\mathrm{M}^{+}+2\right], 80\right), 301(48), 303$ (40), 2666 (48), 91 (100). Exact mass calcd. for $\mathrm{C}_{15} \mathrm{H}_{7} \mathrm{~N}_{2} \mathrm{OCl}_{3}:$ 335.9627. Found: 335.9624 .

3,4,6-trichloro-5-p-nitrophenoxyphthalonitrile (10). Following the procedure described for 8 above, but using 3,4,5,6-tetrachlorophthalonitrile (2), and $p$-nitrophenol (5), a product was obtained which chromatographed on flash grade silica gel using ethyl acetate / hexane (5:95) to get a light brown powder. Recrystallization from hexane gave a light yellow solid (43 mg, 12\%), mp 187-189 ${ }^{\circ} \mathrm{C}$. ${ }^{1} \mathrm{H}$ NMR (400 $\left.\mathrm{MHz} \mathrm{CDCl}_{3}\right): \delta_{\mathrm{H}}, \operatorname{ppm} 6.98(\mathrm{~d}, \mathrm{~J}=9.1 \mathrm{~Hz}, 2 \mathrm{H}), 8.31$ $(\mathrm{d}, \mathrm{J}=9.1 \mathrm{~Hz}, 2 \mathrm{H})$. MS (EI, \%): $\mathrm{m} / \mathrm{z} 367$ ([M]+ $\left.{ }^{+}, 100\right)$, $369\left(\left[\mathrm{M}^{+}+2\right], 95\right), 337(76), 339(72), 286(84), 275$ (72). Exact mass calcd. for $\mathrm{C}_{14} \mathrm{H}_{4} \mathrm{~N}_{3} \mathrm{O}_{3} \mathrm{Cl}_{3}: 366.9321$. Found: 366.9318 
5,7(6,8)-di-tert-butyl-4-p-tert-butylphenoxy-3fluorodibenzo[b,e][1,4]dioxin-1,2-dicarbonitrile (13). Using the procedure described below for 14, but having 6 as the phthalonitrile and 11 as the catechol, a product was obtained which was purified by flash silica gel column chromatography using ethyl acetate/ hexane (1:1) as the eluting solvents to get a powdery material. Re-chromatography with flash grade silica gel using ethyl acetate / hexane / chloroform (1:9:3) gave yellow crystals of 13 (54 mg, 42\%), mp 191$193^{\circ} \mathrm{C}$. ' $\mathrm{H}$ NMR (400 MHz, $\mathrm{CDCl}_{3}$ ): $\delta_{\mathrm{H}}$, ppm 1.25 $(\mathrm{s}, 9 \mathrm{H}), 1.34(\mathrm{~s}, 9 \mathrm{H}), 1.48(\mathrm{~s}, 9 \mathrm{H}), 6.69(\mathrm{~d}, \mathrm{~J}=1.1$ $\mathrm{Hz}, 1 \mathrm{H}), 6.96(\mathrm{~d}, \mathrm{~J}=8.3 \mathrm{~Hz}, 2 \mathrm{H}), 7.07(\mathrm{~d}, \mathrm{~J}=1.1$ $\mathrm{Hz}, 1 \mathrm{H}), 1 \mathrm{H}), 7.39$ (d, J = 8.3 Hz, 2H). MS (EI, \%): $m / z 512$ ([M] $\left.{ }^{*}, 100\right), 513$ (48), 499 (49), 497 (100). Anal. calcd. for $\mathrm{C}_{32} \mathrm{H}_{33} \mathrm{~N}_{2} \mathrm{O}_{3} \mathrm{~F}: \mathrm{C}, 75.01 ; \mathrm{H}, 6.77 ; \mathrm{N}$, 5.41. Found: $\mathrm{C}, 75.03 ; \mathrm{H}, 6.49 ; \mathrm{N}, 5.47 \%$. Exact mass calcd. for $\mathrm{C}_{32} \mathrm{H}_{33} \mathrm{~N}_{2} \mathrm{O}_{3} \mathrm{~F}: 512.2475$. Found: 512.2475.

5,7(6,8)-di-tert-butyl-3-fluoro-4-p-methylphenoxydibenzo[b,e][1,4]dioxin-1,2-dicarbonitrile (14). 3,5-di-tert-butylcatechol (11) (111 mg, 0.5 mmol) in dry DMF solution (8 $\mathrm{mL}$ ) and $\mathrm{K}_{2} \mathrm{CO}_{3}(138$ $\mathrm{mg}, 1 \mathrm{mmol}$ ) was stirred at room temperature under argon for $30 \mathrm{~min}$, causing a reaction in which a green color developed. 3,4,6-trifluoro-5-p-methylphenoxyphthalonitrile (7) (144 mg, $0.5 \mathrm{mmol})$ was added and the reaction was maintained under anhydrous conditions for $20 \mathrm{~h}$ at $45^{\circ} \mathrm{C}$. The solvents were then removed by rotary evaporation at $60^{\circ} \mathrm{C}$, the residue was suspended in ethyl acetate, suction filtered through Celite, and the solvent was removed from the filtrate in vacuo at $40^{\circ} \mathrm{C}$. This brown residue was chromatographed with flash grade silica gel using ethyl acetate / hexane (1:4) as the eluting solvents to get a soft powder. Recrystallization from hexane gave yellow crystals of 14 (169 mg, 72\%), mp 199 $201^{\circ} \mathrm{C}$. ${ }^{1} \mathrm{H} \mathrm{NMR}\left(400 \mathrm{MHz}, \mathrm{CDCl}_{3}\right): \delta_{\mathrm{H}}, \mathrm{ppm} 1.26(\mathrm{~s}$, 9H), $1.48(\mathrm{~s}, 9 \mathrm{H}), 2.37(\mathrm{~s}, 3 \mathrm{H}), 6.70(\mathrm{~d}, \mathrm{~J}=1.1 \mathrm{~Hz}$, $1 \mathrm{H}), 6.93(\mathrm{~d}, \mathrm{~J}=8.0 \mathrm{~Hz}, 2 \mathrm{H}), 7.07(\mathrm{~d}, \mathrm{~J}=1.1 \mathrm{~Hz}, 1 \mathrm{H})$, $7.18(\mathrm{~d}, \mathrm{~J}=8.0 \mathrm{~Hz}, 2 \mathrm{H})$. MS (EI, \%): $m / z 470\left([\mathrm{M}]^{+}\right.$, 88), 455 (100). Anal. calcd. for $\mathrm{C}_{29} \mathrm{H}_{27} \mathrm{~N}_{2} \mathrm{O}_{3} \mathrm{~F}, \%$ : C, $74.22 ; \mathrm{H}, 5.67 ; \mathrm{N}, 6.19$. Found: $\mathrm{C}, 74.02 ; \mathrm{H}, 5.78 ; \mathrm{N}$, 5.96. Exact mass calcd. for $\mathrm{C}_{29} \mathrm{H}_{27} \mathrm{~N}_{2} \mathrm{O}_{3} \mathrm{~F} 470.2001$. Found: 470.2005 .

4-p-tert-butylphenoxy-3-fluoro-5(8)-methoxydibenzo[b,e][1,4]dioxin-1,2-dicarbonitrile (15). Using the procedure described above for 14 , but having 6 as the phthalonitrile and 3-methoxycatechol (12), a crude product was obtained which was purified by flash silica gel column chromatography using ethyl acetate/ hexane (1:1) as the eluting solvents to get a soft powder. Recrystallization from hexane gave a yellow crystalline mixture. Further recrystallization from ethyl acetate / hexane (1:9) yielded light yellow crystals (33 mg, 31\%), mp $175-177^{\circ} \mathrm{C}$. 'H NMR (400 $\left.\mathrm{MHz}, \mathrm{CDCl}_{3}\right): \delta_{\mathrm{H}}, \mathrm{ppm} 1.33(\mathrm{~s}, 9 \mathrm{H}), 3.92(\mathrm{~s}, 3 \mathrm{H}), 6.44$ $(\mathrm{d}, \mathrm{J}=8.5 \mathrm{~Hz}, 1 \mathrm{H}), 6.69(\mathrm{~d}, \mathrm{~J}=8.4 \mathrm{~Hz}, 1 \mathrm{H}), 6.92$ $7.01(\mathrm{~m}, 3 \mathrm{H}), 7.38(\mathrm{~d}, \mathrm{~J}=8.4 \mathrm{~Hz}, 2 \mathrm{H}) . \mathrm{MS}(\mathrm{EI}, \%)$ : $m / z 431\left(\left[\mathrm{M}^{+}+1\right], 15\right), 430\left([\mathrm{M}]^{+}, 48\right), 416(49), 415$ (100). Exact mass calcd. for $\mathrm{C}_{25} \mathrm{H}_{19} \mathrm{~N}_{2} \mathrm{O}_{4} \mathrm{~F}: 430.1334$. Found: 430.1328 .

3-fluoro-5(8)-methoxy-4-p-methylphenoxydibenzo[b,e][1,4]dioxin-1,2-dicarbonitrile (16). Using the procedure described above for 14, but having 7 as the phthalonitrile and 12, gave a crude product, which was recrystallized from ethyl acetate / hexane (1:9) to get light yellow crystals of (16) (267 mg, 69\%), mp 234-236 ${ }^{\circ} \mathrm{C}$. 'H NMR ( $400 \mathrm{MHz}$, $\left.\mathrm{CDCl}_{3}\right): \delta_{\mathrm{H}}, \mathrm{ppm} 2.36(\mathrm{~s}, 3 \mathrm{H}), 3.92(\mathrm{~s}, 3 \mathrm{H}), 6.44(\mathrm{~d}, \mathrm{~J}$ $=8.4 \mathrm{~Hz}, 1 \mathrm{H}), 6.70(\mathrm{~d}, \mathrm{~J}=8.6 \mathrm{~Hz}, 1 \mathrm{H}), 6.90-6.94(\mathrm{~m}$, $3 \mathrm{H}), 7.17(\mathrm{~d}, \mathrm{~J}=7.8 \mathrm{~Hz}, 2 \mathrm{H})$. Exact mass calcd. for $\mathrm{C}_{22} \mathrm{H}_{13} \mathrm{~N}_{2} \mathrm{O}_{4} \mathrm{~F}: 388.0864$. Found: 388.0859 .

5,7(6,8)-di-tert-butyl-4-p-tert-butylphenoxy-3chlorodibenzo[b,e][1,4]dioxin-1,2-dicarbonitrile (17). Using the procedure described above for 14 , but having 8 as the phthalonitrile and 11 , a product was obtained which was purified by flash silica gel column chromatography using ethyl acetate / hexane (1:1) as the eluting solvents to get a soft powder which was recrystal lized from hexane to get light yellow crystals (88 mg, 63\%), mp 264-266 ${ }^{\circ} \mathrm{C}$. 'H NMR (400 MHz, $\left.\mathrm{CDCl}_{3}\right): \delta_{\mathrm{H}}, \mathrm{ppm} 1.02(\mathrm{~s}, 9 \mathrm{H}), 1.27(\mathrm{~s}, 9 \mathrm{H}), 1.29(\mathrm{~s}$, $9 \mathrm{H}), 6.83(\mathrm{~d}, \mathrm{~J}=8.6 \mathrm{~Hz}, 2 \mathrm{H}), 6.93(\mathrm{br} \mathrm{s}, 1 \mathrm{H}), 6.97(\mathrm{br}$ $\mathrm{s}, 1 \mathrm{H}), 7.34(\mathrm{~d}, \mathrm{~J}=8.5 \mathrm{~Hz}, 2 \mathrm{H})$. MS (EI, \%): $\mathrm{m} / \mathrm{z} 536$ $\left(\left[\mathrm{M}^{+}+8\right], 15\right), 534\left(\left[\mathrm{M}^{+}+6\right], 5\right), 532\left(\left[\mathrm{M}^{+}+4\right], 50\right)$, $530\left(\left[\mathrm{M}^{+}+2\right], 48\right), 528\left([\mathrm{M}]^{+}, 97\right), 519(50), 517(25)$, 515 (48), 513 (100). Anal. calcd. for $\mathrm{C}_{32} \mathrm{H}_{33} \mathrm{~N}_{2} \mathrm{O}_{3} \mathrm{Cl}$, \%: C, 72.46; H, 6.46; N, 5.31. Found: C, 72.76; H, $6.30 ; \mathrm{N}, 5.30$. Exact mass calcd. for $\mathrm{C}_{32} \mathrm{H}_{33} \mathrm{~N}_{2} \mathrm{O}_{3} \mathrm{Cl}$ : 528.2184. Found: 528.2179 .

5,7(6,8)-di-tert-butyl-3-chloro-4-p-methylphenoxydibenzo[b,e][1,4]dioxin-1,2-dicarbonitrile (18). Using the procedure described above for 14, but having 9 as the phthalonitrile and 11, a product was obtained which was purified by flash silica gel column chromatography using ethyl acetate / hexane (1:1) as the eluting solvents to give a soft powder. Re-chromatography with flash grade silica gel using ethyl acetate / hexane / chloroform (1:9:3) yielded yellow crystals of $18(42 \mathrm{mg}, 34 \%), \mathrm{mp} 208-210^{\circ} \mathrm{C}$. ${ }^{1} \mathrm{H}$ NMR (400 MHz, $\mathrm{CDCl}_{3}$ ): $\delta_{\mathrm{H}}, \mathrm{ppm} 1.23(\mathrm{~s}, 9 \mathrm{H})$, $1.47(\mathrm{~s}, 9 \mathrm{H}), 2.36(\mathrm{~s}, 3 \mathrm{H}), 6.60(\mathrm{~s}, 1 \mathrm{H}), 6.87$ (br s, 2H), 7.04 (s, 1H), 7.17 (br s, 2H). MS (EI, \%): $\mathrm{m} / \mathrm{z}$ $489\left(\left[\mathrm{M}^{+}+3\right], 50\right), 487\left(\left[\mathrm{M}^{+}+1\right], 100\right), 474(50), 472$ (100). Exact mass calcd. for $\mathrm{C}_{32} \mathrm{H}_{33} \mathrm{~N}_{2} \mathrm{O}_{3} \mathrm{~F}: 486.1697$. Found: 486.1710 .

5,7(6,8)-di-tert-butyl-3,4-dioctanyloxydibenzo[b,e][1,4]dioxin-1,2-dicarbonitrile (19). Dioxin $14(50 \mathrm{mg}, 0.106 \mathrm{mmol})$ in dry DMF $(3 \mathrm{~mL})$ with 1-octanol (56 mg, $0.4 \mathrm{mmol}$ ) and $\mathrm{K}_{2} \mathrm{CO}_{3}(20 \mathrm{mg}$, $0.15 \mathrm{mmol}$ ) were stirred at $100^{\circ} \mathrm{C}$ under an argon atmosphere for $30 \mathrm{~h}$. The solvents were removed to get a light brown solid. This residue was suspended in ethyl acetate, suction filtered through Celite, and 


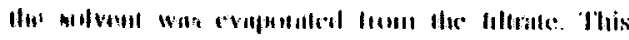

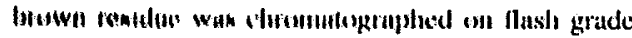
Ition pol tisitus elliyl tceltule / hexune / chloroform (1:9:3) got a llygu yelfow paste in low yield. 'H NMR (400 $\mathrm{MHz}_{2} \mathrm{CDCl}_{3}$ ): $\AA_{\mu}$, ppm 0.91 (br s, 6H), 1.30 ( br s. $9 \mathrm{H}), 1.23-1.28(\mathrm{~m}, 16 \mathrm{H}), 1.45(\mathrm{~s}, 9 \mathrm{H}), 1.45-1.48$ $(\mathrm{m}, 4 \mathrm{H}), 1.82-1.86(\mathrm{~m}, 4 \mathrm{H}), 4.15-4.24(\mathrm{tt}, 4 \mathrm{H}), 6.82$ 7.05 (m, 2H). MS (EI, \%): $m / z 603\left(\left[\mathrm{M}^{+}+1\right], 12\right)$, $602\left([\mathrm{M}]^{+}, 20\right), 490(9), 363(13), 279(17), 167(28)$, $149(64), 85(64), 84(100), 71(71), 57(80), 49(83)$ Exact mass calcd. for $\mathrm{C}_{38} \mathrm{H}_{54} \mathrm{~N}_{2} \mathrm{O}_{4}: 602.4071$. Found: 602.4083 .

$3-[3,5(4,6$,$) -di-tert-butyl-2-hydroxyphenoxy]-6-$ fluoro-5-p-methylphenoxy-3-octanyloxyphthalonitrile (20). Dioxin 14 ( $50 \mathrm{mg}, 0.106 \mathrm{mmol}$ ) in 1 octanol ( $3 \mathrm{~mL}$ ) with $\mathrm{K}_{2} \mathrm{CO}_{3}(20 \mathrm{mg}, 0.15 \mathrm{mmol})$ were stirred at $85^{\circ} \mathrm{C}$ under an argon atmosphere for $72 \mathrm{~h}$. The solvents were removed by rotary evaporation at $80^{\circ} \mathrm{C}$ to get a light brown paste The residue was suspended in ethyl acetate, suction filtered through Celite and the solvent was removed from the filtrate in vacuo at $40^{\circ} \mathrm{C}$. This brown residue was chromatographed on flash grade silica gel using ethyl acetate / hexane / chloroform (1:9:3) to obtain in low yield yellow crystals, mp $195 \sim 197^{\circ} \mathrm{C}$. ${ }^{1} \mathrm{H}$ NMR (400 $\left.\mathrm{MHz}, \mathrm{CDCl}_{3}\right): \delta_{\mathrm{H}}, \operatorname{ppm~} 0.9(\mathrm{t}, \mathrm{J}=6.9 \mathrm{~Hz}, 3 \mathrm{H}), 1.26$ $(\mathrm{s}, 9 \mathrm{H}), 1.23-1.28(\mathrm{~m}, 10 \mathrm{H}), 1.52(\mathrm{~m}, 11 \mathrm{H}), 2.34(\mathrm{~s}$, $3 \mathrm{H}), 4.16(\mathrm{t}, \mathrm{J}=6.6 \mathrm{~Hz}, 2 \mathrm{H}), 6.71(\mathrm{~s}, 1 \mathrm{H}), 6.91(\mathrm{~d}$, $\mathrm{J}=8.0 \mathrm{~Hz}, 2 \mathrm{H}), 7.02(\mathrm{~s}, 1 \mathrm{H}), 7.13(\mathrm{~d}, \mathrm{~J}=8.0 \mathrm{~Hz}$, 2H). MS (EI, \%): $m / z 600\left([\mathrm{M}]^{+}, 100\right), 487(56), 472$ (52). Exact mass calcd. for $\mathrm{C}_{37} \mathrm{H}_{45} \mathrm{~N}_{2} \mathrm{O}_{4} \mathrm{~F}$ : 599.3288 . Found: 599.3285

3,6-dibromo-4,5-dimethoxyphthalonitrile (23). Dibromoisocyanuric acid $[13,14](2.76 \mathrm{~g}, 9.6 \mathrm{mmol})$ was dissolved in $200 \mathrm{~mL}$ of $4.5 \%$ fuming sulfuric acid and cooled using an ice bath for $30 \mathrm{~min}$. $4,5-$ dimethoxyphthalonitrile (21) [15] $(0.9 \mathrm{~g}, 4.8 \mathrm{mmol})$ was added and the mixture stirred for $7 \mathrm{~min}$, after which the reaction mixture was poured onto $1 \mathrm{Kg}$ of ice. The resulting mixture was stirred for $2 \mathrm{~min}$ and extracted 3 times with $30 \mathrm{~mL}$ of diethyl ether. The organic layer was separated, washed with water, $1 \%$ $\mathrm{NaHCO}_{3}$ solution dried over $\mathrm{MgSO}_{4}$, and filtered. Evaporation of the solvent and purification of the residue by flash silica gel chromatography using ethyl acetate / hexane (1:9) as eluant gave in $52 \%$ yield $0.83 \mathrm{~g}$ of crystals of $23, \mathrm{mp} 222-224^{\circ} \mathrm{C}$. IR (KBr): $v, \mathrm{~cm}^{-1} 2226(\mathrm{CN})$. ' $\mathrm{H}$ NMR (400 $\mathrm{MHz}^{2} \mathrm{CDCl}_{3}$ ): $\delta_{\mathrm{H}}$, ppm 4.01 (s, 6H). ${ }^{13} \mathrm{C} \mathrm{NMR}\left(\mathrm{CDCl}_{3}\right): \delta_{\mathrm{C}}$, ppm 155.36, $122.50,116.46,113.49,61.62$. MS (EI, \%): $\mathrm{m} / \mathrm{z} 346$ $\left([\mathrm{M}]^{+}, 100\right)$. Anal. calcd. for $\mathrm{C}_{10} \mathrm{H}_{6} \mathrm{~N}_{2} \mathrm{O}_{2} \mathrm{Br}_{2}, \%$ : C, $34.72 ; \mathrm{H}, 1.75 ; \mathrm{N}, 8.09$. Found: C, 34.95; H, 1.71; N, 8.07 .

3,6-dibromo-4,5-dineopentoxyphthalonitrile (24). To a mixture of $1.43 \mathrm{~g}$ ( $5 \mathrm{mmol}$ ) of dibromoisocyanuric acid in $250 \mathrm{~mL}$ of $2.5 \%$ fuming sulfuric acid at $-10^{\circ} \mathrm{C}$, was added $0.5 \mathrm{~g}(1.7 \mathrm{mmol})$ of 4,5 - dineopcentoxyphthalonitrile (22) [16]. As soon as 22 dissolved, the reaction mixture was poured onto $1 \mathrm{Kg}$ of ice and worked up as desribed above for 23 to give $0.32 \mathrm{~g}$ ( $42 \%$ yield) of $24, \mathrm{mp} 167-169^{\circ} \mathrm{C}$. IR (KBr): $v, \mathrm{~cm}^{-1} 2233(\mathrm{CN})$. 'H NMR (400 $\left.\mathrm{MHz}^{\mathrm{H}} \mathrm{CDCl}_{3}\right): \delta_{\mathrm{H}}$, ppm 1.11 (s 18H), 3.79 (s, 4H). ${ }^{13} \mathrm{C} \mathrm{NMR}\left(\mathrm{CDCl}_{3}\right)$ : $\delta_{\mathrm{C}}$, ppm 154.95, 122.59, 116.19, 113.77, 84.55, 32.83, 25.48. MS (EI, \%): $m / z 458\left([\mathrm{M}]^{+}, 100\right)$. Anal. calcd. for $\mathrm{C}_{18} \mathrm{H}_{22} \mathrm{~N}_{2} \mathrm{O}_{2} \mathrm{Br}_{2}$, \%: C, 47.18; $\mathrm{H}, 4.84 ; \mathrm{N}, 6.11$. Found: C, $47.31 ; \mathrm{H}, 4.87 ; \mathrm{N}, 6.01$.

General procedure for the $\mathrm{Ni}^{\circ}$-coupling reactions of the dibromophthalonitriles 23 and 24 [17]. To a solution of $0.358 \mathrm{mmol}$ of 3.6 -dibromo4,5-dimethoxyphthalonitrile (23) or 3,6-dibromo4,5-dineopentoxyphthalonitrile (24), $0.04 \mathrm{mmol}$ of $\mathrm{Ni}(\mathrm{acac})_{2}, 0.152 \mathrm{mmol}$ of $\mathrm{PPh}_{3}$ and $0.456 \mathrm{mmol}$ of commercially available (Aldrich) tributyl(phenylethynyl)tin or tributylvinyltin in $4 \mathrm{~mL}$ of dry tetrahydrofuran (THF), was added $0.25 \mathrm{~mL}$ of $0.95 \mathrm{M}$ hexane solution of disobutylaluminum hydride (DIBAH) and the mixture was stirred at $85^{\circ} \mathrm{C}$. After $12 \mathrm{~h}$ a second $0.025 \mathrm{~mL}$ of DIBAH was added and heated for $20 \mathrm{~h}$. For 24, after the first DIBAH addition, the solution was heated for $16 \mathrm{~h}$, after the second addition for $32 \mathrm{~h}$ and a third addition for $10 \mathrm{~h}$. The reaction mixture was cooled to room temperature, 3

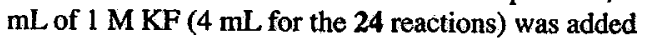
and the mixture stirred for $1 \mathrm{~h}$. The precipitate was filtered and washed three times with $50 \mathrm{~mL}$ of diethyl ether. The ether layer was successively washed with water, brine and dried over anhydrous $\mathrm{Na}_{2} \mathrm{SO}_{4}$. Flash silica gel chromatography of the product using benzene (Caution: carcinogen) as solvent gave three products for each reaction, the desired bis-coupled product, a mono-coupled product, and debrominated products as listed below.

4,5-dimethoxy-3,6-bisphenylethynylphthalonitrile (27). Phthalonitrile 27 (54 mg) was obtained in $54 \%$ yield as crystals, $\mathrm{mp} 148-150^{\circ} \mathrm{C}$. IR (KBr): $v, \mathrm{~cm}^{* 1} 2233(\mathrm{CN}) .{ }^{1} \mathrm{H}$ NMR (400 $\mathrm{MHz}, \mathrm{CD}_{2} \mathrm{Cl}_{2}$ ): $\delta_{\mathrm{H}}$, ppm $4.19(\mathrm{~s}, 6 \mathrm{H}), 7.46-7.51(\mathrm{~m}, 6 \mathrm{H}), 7.68-7.70(\mathrm{~m}$, $4 \mathrm{H})$. MS (EI, \%): $m / z 388\left([\mathrm{M}]^{+}, 100\right)$. Anal. calcd. for $\mathrm{C}_{26} \mathrm{H}_{16} \mathrm{~N}_{2} \mathrm{O}_{2}, \%: \mathrm{C}, 80.40 ; \mathrm{H}, 4.15 ; \mathrm{N}, 7.21$. Found: $\mathrm{C}, 80.42 ; \mathrm{H}, 4.13 ; \mathrm{N}, 7.22$.

3-bromo-4,5-dimethoxy-6-phenylethynylphthalonitrile (28). Phthalonitrile $28(27.5 \mathrm{mg})$ was obtained in $21 \%$ yield as crystals, mp $185-186^{\circ} \mathrm{C}$. IR (KBr): $v, \mathrm{~cm}^{-1} 2226(\mathrm{CN})$. ${ }^{1} \mathrm{H}$ NMR (400 MHz, $\left.\mathrm{CD}_{2} \mathrm{Cl}_{2}\right): \delta_{\mathrm{H}}$ ppm $4.04(\mathrm{~s}, 3 \mathrm{H}), 4.20(\mathrm{~s}, 3 \mathrm{H}), 7.45-$ $7.51(\mathrm{~m}, 3 \mathrm{H}), 7.67-7.69(\mathrm{~m}, 2 \mathrm{H})$. MS (EI, \%): $\mathrm{m} / \mathrm{z}$ $366\left([\mathrm{M}]^{+}, 100\right)$. Anal. calcd. for $\mathrm{C}_{18} \mathrm{H}_{11} \mathrm{BrN}_{2} \mathrm{O}_{2}, \%$ : C, 58.88; H, 3.02; N, 7.63. Found: C, $58.83 \mathrm{H}, 3.07$; N, 7.59 .

4,5-dimethoxy-3-phenylethynylphthalonitrile (29). Phthalonitrile 29 (19.6 mg) was obtained in $19 \%$ yield as crystals, mp $130-131^{\circ} \mathrm{C}$. IR (KBr): $v, \mathrm{~cm}^{-1}$ 2233 (CN). 'H NMR (400 $\mathrm{MHz}, \mathrm{CD}_{2} \mathrm{Cl}_{2}$ ): $\delta_{\mathrm{H}} \mathrm{ppm}$ 
$3.98(\mathrm{~s}, 3 \mathrm{H}), 4.11(\mathrm{~s}, 3 \mathrm{H}), 7.20,(\mathrm{~s}, 1 \mathrm{H}) 7.40-7.41(\mathrm{~m}$, $3 \mathrm{H}), 7.63-7.65(\mathrm{~m}, 2 \mathrm{H})$. MS (EI, \%): $\mathrm{m} / \mathrm{z} 288\left([\mathrm{M}]^{+}\right.$, 100). Anal. calcd. for $\mathrm{C}_{18} \mathrm{H}_{12} \mathrm{~N}_{2} \mathrm{O}_{2}, \%: \mathrm{C}, 74.99 ; \mathrm{H}$, $4.20 ; \mathrm{N}, 9.72$. Found: $\mathrm{C}, 74.87, \mathrm{H}, 4.26 ; \mathrm{N}, 9.81$.

4,5-dimethoxy-3,6-divinylphthalonitrile (30). Phthalonitrilc 30 ( $24 \mathrm{mg}$ ) was obtained in $28 \%$ yield as crystals, mp $98-100^{\circ} \mathrm{C}$. IR (KBr): $\nu, \mathrm{cm}^{-1} 2257$ (CN). 'H NMR (400 MHz, $\mathrm{CDCl}_{3}$ ): $\delta_{\mathrm{H}} \mathrm{ppm} 5.82-$ $5.85(\mathrm{~m}, 2 \mathrm{H}), 6.26-6.30(\mathrm{~m}, 2 \mathrm{H}), 6.85-6.92(\mathrm{~m}, 2 \mathrm{H})$. MS (EI, \%): $m / z: 240[\mathrm{M}]^{+}, 225,197$. Anal. calcd. for $\mathrm{C}_{14} \mathrm{H}_{12} \mathrm{~N}_{2} \mathrm{O}_{2}$, \%: $\mathrm{C}, 69.99 ; \mathrm{H}, 5.03 ; \mathrm{N}, 11.06$. Found: C, $70.04 ; \mathrm{H}, 5.08 ; \mathrm{N}, 11.57$.

3-bromo-4,5-dimethoxy-6-vinylphthalonitrile (31). Phthalonitrile $31(21 \mathrm{mg})$ was obtained in $20 \%$ yield as crystals, mp $145-146^{\circ} \mathrm{C}$. IR (KBr): $\nu, \mathrm{cm}^{-1}$ $2240(\mathrm{CN})$. ' $\mathrm{H}$ NMR $\left(400 \mathrm{MHz}, \mathrm{CDCl}_{3}\right): \delta_{\mathrm{H}} \mathrm{ppm}$ $3.95(\mathrm{~s}, 3 \mathrm{H}), 4.03(\mathrm{~s}, 3 \mathrm{H}), 5.82-5.89(\mathrm{~m}, 1 \mathrm{H}), 6.25-$ $6.31(\mathrm{~m}, 1 \mathrm{H}), 6.81-6.92(\mathrm{~m}, 1 \mathrm{H})$. MS (EI, \%): $\mathrm{m} / \mathrm{z}$ $292[\mathrm{M}]^{+}, 277,198$. Anal. calcd. for $\mathrm{C}_{12} \mathrm{H}_{9} \mathrm{BrN}_{2} \mathrm{O}_{2}, \%$ : C, 49.17; H, 3.09; N, 9.56. Found: C, 49.09; H, 3.06; N, 9.49 .

4,5-dimethoxy-3-vinylphthalonitrile (32). Phthalonitrile $32(12.5 \mathrm{mg})$ was obtained in $16 \%$ yield as crystals, mp $139-140^{\circ} \mathrm{C}$. IR (KBr): $v, \mathrm{~cm}^{-1} 2260$ (CN). 'H NMR (400 MHz, $\mathrm{CDCl}_{3}$ ): $\delta_{\mathrm{H}}$ ppm $3.90(\mathrm{~s}$, $3 \mathrm{H}), 3.99(\mathrm{~s}, 3 \mathrm{H}), 5.81-5.84(\mathrm{~m}, 1 \mathrm{H}), 6.22-6.26(\mathrm{~m}$, $1 \mathrm{H}), 6.85-6.92(\mathrm{~m}, 1 \mathrm{H})$. MS (EI, \%): $m / z 214[\mathrm{M}]^{+}$, 199,171 . Anal. calcd. for $\mathrm{C}_{12} \mathrm{H}_{10} \mathrm{~N}_{2} \mathrm{O}_{2}, \%: \mathrm{C}, 67.28 ; \mathrm{H}$, $4.71 ; \mathrm{N}, 13.08$. Found: C, 67.34; H, 4.78; N, 13.46 .

4,5-dineopentoxy-3,6-bisphenylethynylphthalonitrile (33). Phthalonitrile 33 (66 mg) was obtained in $35 \%$ yield as crystals, $\mathrm{mp} 115-116^{\circ} \mathrm{C}$. IR $(\mathrm{KBr}): v$, $\mathrm{cm}^{-1} 2218(\mathrm{CN})$. ' $\mathrm{H} \mathrm{NMR}\left(400 \mathrm{MHz}, \mathrm{CDCl}_{3}\right.$ ): $\delta_{\mathrm{H}} \mathrm{ppm}$ $1.12(\mathrm{~s}, 18 \mathrm{H}) 3.95(\mathrm{~s}, 4 \mathrm{H}), 7.39-7.42(\mathrm{~m}, 6 \mathrm{H}), 7.62-$ $7.63(\mathrm{~m}, 4 \mathrm{H})$. MS (EI, \%): $m / z 500[\mathrm{M}]^{+}, 360,340$, 260, 105. Anal. calcd. for $\mathrm{C}_{34} \mathrm{H}_{32} \mathrm{~N}_{2} \mathrm{O}_{2}, \%$ : C, 81.57; $\mathrm{H}, 6.44 ; \mathrm{N}, 5.60$. Found: C, 81.50; H, 6.50; N, 5.63.

3-bromo-4,5-dineopentoxy-6-phenylethynylphthalonitrile (34). Phthalonitrile $34(31 \mathrm{mg})$ was obtained in $17 \%$ yield as crystals, $\mathrm{mp} 147-149^{\circ} \mathrm{C}$. IR (KBr): $v, \mathrm{~cm}^{-1} 2231$ (CN). 'H NMR (400 MHz, $\mathrm{CDCl}_{3}$ ): $\delta_{\mathrm{H}} \mathrm{ppm} 1.10(\mathrm{~s}, 9 \mathrm{H}), 1.13(\mathrm{~s}, 9 \mathrm{H}), 3.99$ (s, $2 \mathrm{H}), 4.01(\mathrm{~s}, 2 \mathrm{H}), 7.44-7.46(\mathrm{~m}, 3 \mathrm{H}), 7.59-7.62(\mathrm{~m}$, 2H). MS (EI, \%): $478[\mathrm{M}]^{+}, 378,279,149$. Anal. calcd. for $\mathrm{C}_{26} \mathrm{H}_{27} \mathrm{BrN}_{2} \mathrm{O}_{2}, \%: \mathrm{C}, 65.14 ; \mathrm{H}, 5.68 ; \mathrm{N}$, 5.84. Found: C, $65.11 \mathrm{H}, 5.73 ; \mathrm{N}, 5.82$.

\section{RESULTS AND DISCUSSION}

Tetrasubstituted phthalocyanines can be prepared in one strategy using nucleophilic aromatic substitution reactions on a suitable commercially available tetrasubstituted phthalonitrile such as 3,4,5,6tetrafluorophthalonitrile (1). Although substitution reactions on 1 have been shown before $[11,18]$, to give pure tetra- [11] and monosubstitution [18]

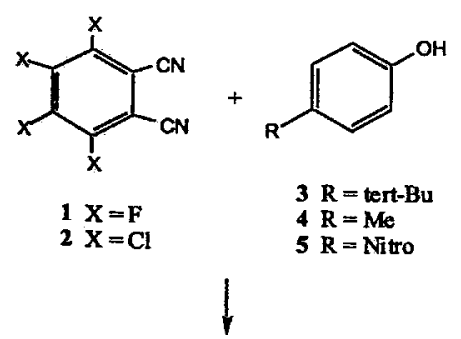<smiles>[R]c1ccc(Oc2c([X])c([X])c(C#N)c(C#N)c2[X])cc1</smiles>

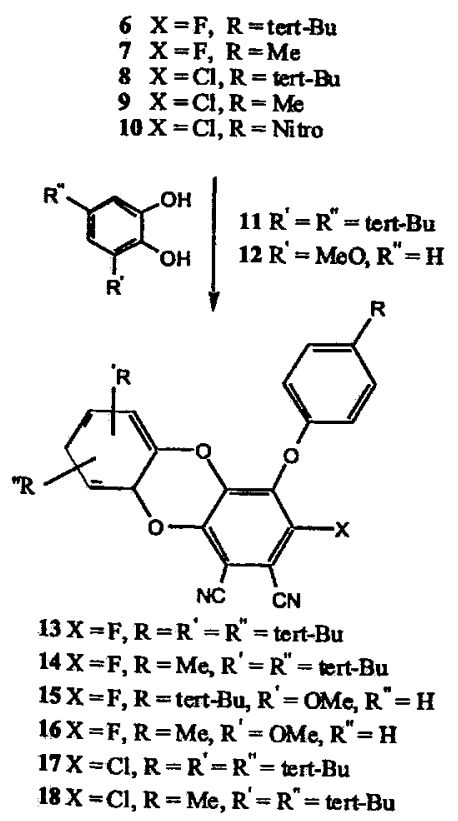

Scheme 1.

products, stepwise selective substitution of the four fluoro substituents are unknown. The difficulty with this approach is the fact that mixtures of products that are difficult to separate may result. However, judicial selection of appropriate nucleophiles can give pure products. Thus, treatment of 1 or $3,4,5,6-$ tetrachlorophthalonitrile (2) with p-tert-butylphenol (3), p-methylphenol (4), or $p$-nitrophenol (5) in DMF and $\mathrm{K}_{2} \mathrm{CO}_{3}$ at $40^{\circ} \mathrm{C}$ for $16 \mathrm{~h}$ gave the $3,4,6$-trihalo5 -phenoxyphthalonitriles $6-10$ in $42,67,43,62$, and $12 \%$ yields respectively (Scheme 1). At higher temperatures disubstitution at the 4 and 5 positions took place.

Subsequently, treatment of 3,5-di-tert-butyl catechol (11) or 3-methoxycatechol (12) with $\mathrm{K}_{2} \mathrm{CO}_{3}$ 


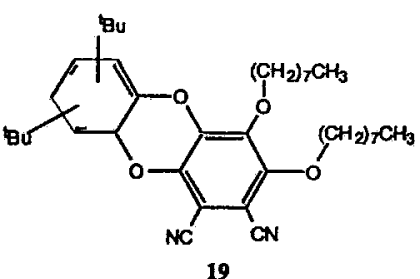

19

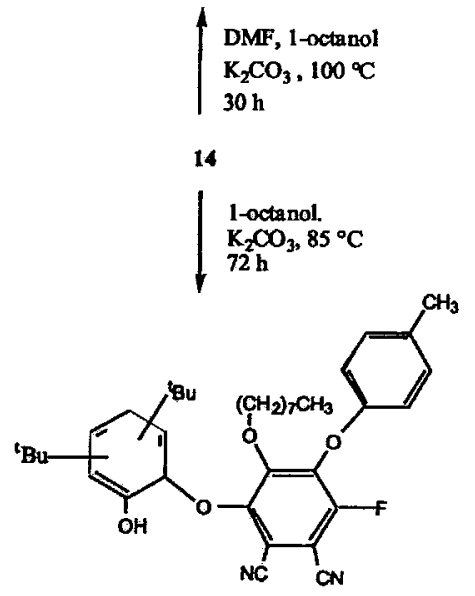

20

Scheme 2 .

in DMF for $30 \mathrm{~min}$ followed by the addition of of 6-7 and further heating for $20 \mathrm{~h}$ at $45^{\circ} \mathrm{C}$ gave the fluorodioxins 13-16 respectively in $42,72,31$, and $69 \%$ yields.

Similar treatment of 9 with 11 and 12 gave the chloro dioxins 17 and 18 in 63 and $34 \%$ yields respectively (Scheme 1). It should be noted that dioxins 13-18 are mixtures of isomers with respect to the tert-butyl or methoxy groups on the dioxin ring. The dioxin1,2 dicarbonitriles 13-18 do represent a rare class of phthalonitiles having four different substituents. We were still interested, however, in replacing the fourth fluoro group by a different substituent. Towards this goal dioxin 14 was treated with 1-octanol in DMF at $100{ }^{\circ} \mathrm{C}$ with $\mathrm{K}_{2} \mathrm{CO}_{3}$ for $30 \mathrm{~h}$ (Scheme 2).

Surprisingly, not only was the fluoro group replaced under these conditions, but the $p$-methylphenoxy group at position 4 was also replaced to give the dioxin 19 containing two octanyloxy substituents (Scheme 2). Under somewhat milder conditions 14 yielded a ring opened product 20 in which the catechol (also a phenoxy group) was displaced (Scheme 2). This important observation should alert one to the possibility that phenoxy groups can be labile, not only in phthalonitriles, but also in phthalocyanine formation when phenoxy substituents are present and<smiles>[R6]c1cc(C#N)c(C#N)cc1O</smiles>

$21 \mathrm{R}=\mathrm{CH}_{3}$ $22 \mathrm{R}=\mathrm{CH}_{2} \mathrm{C}\left(\mathrm{CH}_{3}\right)_{3}$

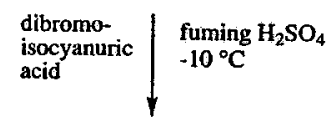<smiles>N#Cc1c(Br)c([18O])c([18O])c(Br)c1C#N</smiles>

$23 \mathrm{R}=\mathrm{CH}_{3}$ $24 \mathrm{R}=\mathrm{CH}_{2} \mathrm{C}\left(\mathrm{CH}_{3}\right)_{3}$

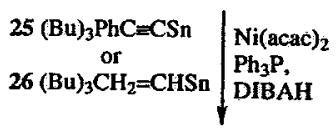<smiles>[R]Oc1c([R])c(C#N)c(C#N)c(P)c1[2H]</smiles>

$27 \mathrm{R}=\mathrm{CH}_{3}, \mathrm{R}^{\prime}=\mathrm{R}^{\prime \prime}=\mathrm{PhC}=\mathrm{C}$ $28 \mathrm{R}=\mathrm{CH}_{3}, \mathrm{R}^{\prime}=\mathrm{PhC}=\mathrm{C}, \mathrm{R}^{\prime \prime}=\mathrm{Br}$ $29 \mathrm{R}=\mathrm{CH}_{3}, \mathrm{R}^{\prime}=\mathrm{PhC}=\mathrm{C}, \mathrm{R}^{\prime \prime}=\mathrm{H}$ $30 \mathrm{R}=\mathrm{CH}_{3}, \mathrm{R}^{\prime}=\mathrm{R}^{\prime \prime}=\mathrm{CH}_{2}=\mathrm{CH}$ $31 \mathrm{R}=\mathrm{CH}_{3}, \mathrm{R}^{\prime}=\mathrm{CH}_{2}=\mathrm{CH}, \mathrm{R}^{\prime \prime}=\mathrm{Br}$ $32 \mathrm{R}=\mathrm{CH}_{3}, \mathrm{R}^{\prime}=\mathrm{CH}_{2}=\mathrm{CH}, \mathrm{R}^{\prime \prime}=\mathrm{H}$ $33 \mathrm{R}=\mathrm{CH}_{2} \mathrm{C}\left(\mathrm{CH}_{3}\right)_{3}, \mathrm{R}^{\prime}=\mathrm{R}^{\prime \prime}=\mathrm{PhC}=\mathrm{C}$ $34 \mathrm{R}=\mathrm{CH}_{2} \mathrm{C}\left(\mathrm{CH}_{3}\right)_{3}, \mathrm{R}^{\prime}=\mathrm{PhC}=\mathrm{C}, \mathrm{R}^{\prime \prime}=\mathrm{Br}$ $22 \mathrm{R}=\mathrm{CH}_{2} \mathrm{C}\left(\mathrm{CH}_{3}\right)_{3}, \mathrm{R}^{\prime}=\mathrm{R}^{\prime \prime}=\mathrm{H}$

\section{Scheme 3.}

bases are used in phthalocyanine synthesis.

Another strategy for preparing multisubstituted phthalocyanines involves performing electrophilic aromatic substutution reactions on unsubstituted phthalonitriles. We have previously shown how phthalonitrile itself can be brominated to give a separable mixture consisting mostly of mono and dibromophthalonitriles [13]. Extending this strategy to the bromination of known alkoxysubstituted phthalonitriles should give polybrominated products which may be further converted to polysubstituted phthalonitriles. It should be noted that alkoxy substituents should activate the phthalonitrile towards polybromination. Thus, addition of 4,5dimethoxyphthalonitrile (21) [15] or 4,5-dineopent oxyphthalonitrile (22) [16] (for an additional seven $\mathrm{min}$ ) to dibromoisocyanuric acid [13,14] (sometimes 
called dibromocyanuric acid), having been dissolved in $4.5 \%$ fuming sulfuric acid at $-10^{\circ} \mathrm{C}$ for $30 \mathrm{~min}$, gave 3,6-dibromo-4,5-dimethoxyphthalonitrile (23) and 3,6-dibromo-4,5-dineopentoxyphthalonitrile (24) in 52 and $42 \%$ yields respectively (Scheme 3 ). In the reaction with 22 only $2.5 \%$ fuming sulfuric acid was used. Too high a percent of fuming sulfuric acid gives lower yields and decomposition products, while too low a percent sulfuric acid gives monobromination or unreacted starting material. We were interested in further coupling of the dibromophthalonitriles 23 and 24 with alkynes and vinyl groups.

Although a vast array of organometalic coupling reagents are possible $[19,20]$, the combination of having a hindered aromatic bromide constrained us to using a method developed by Shirakawa et al. [17] using a $\mathrm{Ni}(\mathrm{acac})_{2}$ catalyst and alkynyltin or vinyltin organometalic reagents (Scheme 3 ).

Thus treatment of 23 or 24 with tri- $n$-butylphenylethynyltin (25) and tri- $n$-butylvinyltin (26) with $\mathrm{Ni}(\mathrm{acac})_{2}$, and triphenylphosphine in THF, followed by diisobutylaluminum hydride (DIBAH) in THF usually gave three products for each reaction, the dicoupled product, the monocoupled product and a reduced product, whereby a bromine group was replaced by hydrogen. This reduction is a wellknown by-product in these coupling reactions. Thus reaction of the dimethoxy phthalonitrile 23 with the phenylethynyltin 25 gave the dicoupled product 27 , the monocoupled product 28 and a reduced product 29 , while the use of the vinyltin 26 in the same reaction yielded the dicoupled product $\mathbf{3 0}$, the monocoupled product 31, and a reduced product 32 . Similarly, reaction of the dineopentoxyphthalonitrile 23 with 25 gave the dicoupled product 33 , the monocoupled product 34 and a reduced product 22 (Scheme 3 ).

\section{CONCLUSION}

We have demonstrated the possibility of synthesizing phthalonitriles having four different substituents in the cases of 13-18 using the protocol of dispacing sequentially fluoro or chloro substituents of 1 and 2 respectively, although the use of fluoro 1 is preferable. One important outcome of this study shows that phenoxy substituents are labile in nucleophilic aromatic substitution reactions. In a second strategy, bromination of alkoxy substituted phthalonitriles can give brominated phthalonitriles, which through subsequent coupling reactions led to tetrasubstitued phthalonitriles having three different substituents, such as $\mathbf{2 7 - 3 4}$. It should be noted that, starting with an unsymmetrical dialkoxyphthalonitrile, phthalonitriles, having four different substituents, could also be made by this route. Further research on improving the yields of these phthalonitriles for subsequent phthalocyanine synthesis is in progress.

\section{Acknowledgements}

The authors are grateful to the Natural Sciences and Engineering Research Council of Canada for support of this reasearch.

\section{REFERENCES}

1. Leznoff $\mathrm{CC}$ and Lever ABP, Eds. Phthalocyanines: Properties and Applications; Wiley-VCH: New York, 1989-1996; Vol. 1-4.

2. McKeown NB. Phthalocyanine Materials: Synthesis, Structure and Function; Cambridge University Press: London, 1998.

3. Mikhalenko SA and Luk'yanets EA. J. Gen Chem USSR 1969; 39: 2081-2086.

4. Hanack M, Metz, J and Pawlowski. G. Chem. Ber. 1982; 115: 2836-2853.

5. Snow AW and Jarvis NL. J. Am. Chem. Soc. 1984; 106: 4706-4711.

6. Leznoff CC, Marcuccio SM, Greenberg S, Lever ABP and Tomer KB. Can. J. Chem. 1985; 63: 623-632.

7. Cook MJ, Daniel MF, Harrison KJ, McKeown NB and Thompson AJ. J. Chem. Soc. Chem. Commun. 1987: 1086-1088.

8. Pawlowski G and Hanack M. Synthesis 1980: 287-289.

9. Piechocki C and Simon J. Nouv. J. Chem. 1985; 9: 159-166.

10. Duggan PJ and Gordon PF. Eur. Pat. Appl. 1985: EP155780.

11. Eberhardt W and Hanack M. Synthesis 1997: 95-100.

12. Bhardwaj N, Andraos J and Leznoff CC. Can. J. Chem. 2002; 80: 141-147.

13. Leznoff CC, Li Z, Isago, H, D'Ascanio AM and Terekhov DS. J. Porphyrins Phthalocyanines. 1999; 3: 406-416.

14. Gottardi W. Monatsh. Chem. 1968; 99: 815-

15. Metz J. Schneider $O$ and Hanack M. Inorg. Chem. 1984; 23: 1065-1071.

16. Khanamiryan AKh, Bhardwaj $\mathrm{N}$ and Leznoff CC. J. Porphyrins Phthalocyanines 2000; 4 : 484-490.

17. Shirakawa E, Yamasaki, $K$ and Hiyama $T$. Synthesis 1998: 1544-1549.

18. Birchall JM, Haszeldine RN and Morley JO. $J$. Chem. Soc. (C) 1970: 456-462.

19. Littke AF and Fu GC. Angew. Chem. Int. Ed. Engl. 2002; 41: 4176-4211.

20. Stille JK. Angew. Chem. Int. Ed. Engl. 1986; 25: 508-524. 